\title{
Abkürzungen von Autorenverbänden und literarischen Vereinigungen
}

Die Kürzel D, DDR, A, CH und YA (internationale Autorenverbände und weitere literarische Vereinigungen) bezeichnen die Rubriken, unter denen diese Institutionen im entsprechenden Anhang zu finden sind.

\begin{tabular}{|c|c|}
\hline $\mathrm{ADA}$ & Arbeitsgemeinschaft deutschsprachiger Autoren (D) \\
\hline AdK & Akademie der Künste (D) \\
\hline AEK & Arbeitskreis Egerländer Kulturschaffender e. V. (D) \\
\hline AGAV & Arbeitsgemeinschaft alternativer Verlage und Autoren e. V. \\
\hline AGK & Autorengruppe Koblenz (D) \\
\hline AKJ & Arbeitskreis für Jugendliteratur e. V. (D) \\
\hline AKL & Der Autorenkreis Linz \\
\hline AKM & $\begin{array}{l}\text { Staatlich genehmigte Gesellschaft der Autoren, Komponisten und } \\
\text { Musikverleger (A) }\end{array}$ \\
\hline AKV & $\begin{array}{l}\text { Schweizer Gesellschaft volkstümlicher Autoren, Komponisten \& } \\
\text { Verleger }\end{array}$ \\
\hline ALAI & Association Littéraire et Artistique Internationale (IA) \\
\hline ALG e.V. & Arbeitsgemeinschaft literarischer Gesellschaften e. V. (D) \\
\hline ARGE Literatur & $\begin{array}{l}\text { Arbeitsgemeinschaft Literatur im NdÖsterr. Bildungs- und Hei- } \\
\text { matwerk }\end{array}$ \\
\hline ASEM & Schweizerische Schriftstellerärzte-Vereinigung \\
\hline ASTI-SÜDV & Schweizerischer Übersetzer- und Dolmetscherverband \\
\hline ASTL-SVLÜ & Schweizerischer Verband Literarischer Übersetzer \\
\hline ATM 81 & Autorentreff Meissner 81 \\
\hline AUP & Autorenkreis Plesse (IA) \\
\hline AWA & $\begin{array}{l}\text { Anstalt zur Wahrung der Aufführungsrechte auf dem Gebiete der } \\
\text { Musik (DDR) }\end{array}$ \\
\hline AWL & Arbeitsgemeinschaft wissenschaftliche Literatur e. V. (D) \\
\hline AWS & Arbeitsgemeinschaft Werkstatt „schreiben" (D) \\
\hline B.A. & Bundesverband Deutscher Autoren e. V. (D) \\
\hline Ba.S.V. & Basler Schriftsteller-Verein \\
\hline BAV & Bayerische Autorenvereinigung e. V. \\
\hline BBA & Berliner Bibliophilen-Abend (D) \\
\hline BDSÄ & Bundesverband deutscher Schriftsteller-Ärzte e. V. (D) \\
\hline BDÜ & Bundesverband der Dolmetscher und Übersetzer e. V. (D) \\
\hline Be.S.V. & Berner Schriftsteller-Verein \\
\hline BGK & Bibliophilen-Gesellschaft Köln \\
\hline BJA & Bundesring junger Autoren \\
\hline BJV & Bayerischer Joumalistenverband \\
\hline B.St.H. & Bund steirischer Heimatdichter (A) \\
\hline BVJA & Bundesverband junger Autoren und Autorinnen e. V. (D) \\
\hline $\mathrm{CdB}$ & Club der Begegnung (A) \\
\hline CISAC & $\begin{array}{l}\text { Confédération Internationale de Sociétés d'Auteurs et Composi- } \\
\text { teurs }\end{array}$ \\
\hline Concordia & Presseclub Concordia (A) \\
\hline CSG & Carl-Schirren-Gesellschaft e. V. (D) \\
\hline CZG & Carl-Zuckmayer-Gesellschaft e. V. (D) \\
\hline DAR & Deutscher Autorenrat (I \\
\hline DAI & Deutscher Autoren Verband e. V. (D) \\
\hline DF & Deutschfreib \\
\hline
\end{tabular}




dg
dju
DJV
DJW
DKBD
DSSV
DSV
DTV
D.U.

EDFG e. $V$. éditions trèves e.V.

ELK

Elka Club

FDA

F.D.B.

FIT

FRG

F.St.Graz

\section{GAV \\ GdB \\ GDBA \\ G.dr.S.}

GdSL

GEMA

GfdS
GFG
GFP
GGG
GO
GP
GRUR

G.S.D.

GUA

GvlF-Gesellschaft

\section{HHG}

IAD

IADG

IADM

IAP

IBBY

ICW

IDI

IFJ

IG Autoren

IGdA

IJA

IKG

ILG

ISA
Dramaturgische Gesellschaft e. V. (D)

Deutsche Journalisten-Union (D)

Deutscher Journalisten-Verband e. V. (D)

Deutsches Jugendschriftenwerk e. V. (D)

Demokratischer Kulturbund der Bundesrepublik Deutschland

Deutschschweizerischer Sprachverein

Deutscher Schriftstellerverband

Deutscher Textdichter-Verband e. V. (D)

Dramatiker-Union e. V. (D)

Erster Deutscher Fantasy Club e. V. (D)

Verein zur Förderung der künstlerischen Tätigkeiten éditions

trèves e. V. (D)

Ernster Lyrik Kreis (D)

Club für Literatur und Kunst $(\mathrm{CH})$

Freier Deutscher Autorenverband e. V. (D)

Freundeskreis Düsseldorfer Buch '75 e.V.

Fédération Internationale des Traducteurs (IA)

Fritz-Reuter-Gesellschaft e.V. (D)

Forum Stadtpark Graz

Grazer Autorenversammlung

Gesellschaft der Bibliophilen e. V. (D)

Genossenschaft Deutscher Bühnen-Angehöriger (D)

Genossenschaft dramatischer Schriftsteller und Komponisten in

Wien

Gesellschaft für Sprache und Literatur $(\mathrm{CH})$

Gesellschaft für musikalische Aufführungs- und mechanische Ver-

vielfältigungsrechte (D)

Gesellschaft für deutsche Sprache e. V. (D)

Gustav-Freytag-Gesellschaft e. V. (D)

Gesellschaft für Freie Publizistik e. V. (D)

Gesellschaft für Geistesgeschichte (D)

Schweizer Autoren Gruppe Olten

Gruppe Poesie (D)

Deutsche Vereinigung für gewerblichen Rechtsschutz und Urheberrecht e.V. (D)

Gesellschaft Schweizerischer Dramatiker

Gruppe Unnaer Autoren (D)

Gertrud von le Fort-Gesellschaft zur Förderung christlicher Literatur e. V. (D)

Heinrich-Heine-Gesellschaft e. V. (D)

Internationale Autorenrunde Dietrichsblatt (IA)

Internationale Alfred Döblin-Gesellschaft (IA)

Internationale Association Deutschsprachiger Medien e. V. (IA)

Internationales Autoren-Progressiv (IA)

Internationales Kuratorium für das Jugendbuch/International

Board on Books for Young People (IA)

International Council of Women (IA)

Internationales Dialekt Institut

International Federation of Journalists (IA)

Interessengemeinschaft österreichischer Autoren

Interessengemeinschaft deutschsprachiger Autoren e. V. (D)

Initiative Junger Autoren e. V. (D)

Innviertler Künstlergilde (A)

Internationale Lenau-Gesellschaft (IA)

Interessenverband Schreibender Frauen, Autorinnen und Wissenschaftlerinnen (D) 
ISDS

ISV

JAR

$\mathrm{Kg}$.

KLK

KMG

Köla

Kogge

LCB

lgo

LIT

Literaria Eutin

LO

L.S.V.

LU

L.V.G.

LVW

LWG

LWK

Mf.O

MKG

MLB

MÖB

NA

NGL

NLG

ÖKU

Ö.S.V.

P.E.N.

RFFU

RSGI

SAV

S.d.a.R. e.V.

SGAS

SGTK

SIU

SKV

SKWS

slg

SOSV

SSB

SSV

STB

SV-DDR

SZ

TB

TELI

Übersetzergemein-

schaft

UMEM

UNIVERSITAS
Internationaler Schutzverband deutschsprachiger Schriftsteller (IA) Innerschweizer Schriftstellerverein

Journalistischer Arbeitsring e. V. (D)

Die Künstlergilde e. V. (D)

Kronenburger Literatur-Kreis

Karl-May-Gesellschaft e. V. (D)

Klub österreichischer Literaturfreunde und Autoren

Europäische Autorenvereinigung „Die Kogge“e. V. (D)

Literarisches Colloquium Berlin e. V. (D)

Literarische Gruppe Osnabrück e. V.

Literaturzentrum e. V. (D)

Eutiner Literarische Gesellschaft (D)

Lyrischer Oktober (D)

Lëtzebuerger Schrëftstellerverband (IA)

Literarische Union e. V.

Staatlich genehmigte Literarische Verwertungsgesellschaft (A)

Literarische Vereinigung Winterthur $(\mathrm{CH})$

Literarische Werkstatt Gelsenkirchen

Literarische Werkstatt Kreuzberg (D)

Mundartfreunde Österreichs

Mühlviertler Künstlergilde (A)

Münchner Literaturbüro - Haidhauser Werkstatt e. V.

Musischer Ring Osterreich-Baiern (A)

Niedersächsische Autorenhilfe

Neue Gesellschaft für Literatur e. V. 1. Berlin; 2. Erlangen

Neue Literarische Gesellschaft e. V. (D)

Österreichische Künstler-Union

Österreichischer Schriftstellerverband

Poets, Playwrights, Essayists, Editors and Novelists

Rundfunk-Fernseh-Film-Union (D)

Regensburger Schriftstellergruppe International (IA)

Südtiroler Autorenvereinigung (IA)

Schule der anderen Realität e. V. (D)

Society for German-American Studies (IA)

Schweizerische Gesellschaft für Theaterkultur

Schweizerische Journalisten-Union

Salzburger Kulturvereinigung

Stiftung Kulturwerk Schlesien (D)

Surwolder Literaturgespräche (D)

Solothurner Schriftstellerverein $(\mathrm{CH})$

Steirischer Schriftstellerbund

1. Podium 70, Salzburger Schriftstellervereinigung; 2. Schweizerischer Schriftstellerinnen- und Schriftstellerverband

Stelzhamerbund der Freunde oböst. Mundartdichtung im ObÖst.

Volksbildungswerk

Schriftstellerverband der DDR

Deutsches Senryu-Zentrum (D)

Der Turmbund (A)

Technisch-Literarische Gesellschaft e. V.

Interessengemeinschaft von Übersetzern literarischer und wissenschaftlicher Werke (A)

Union Mondiale des Escrivains Médicins

Österreichischer Übersetzer- und Dolmetscherverband ,Universitas" 
VdAiA

VdEJ

VDHLK

VDJ

VDKSÖ

VDSK

V.dt.K.

VdÜ

VFF

VFS

VG Bild-Kunst

V.G.S.

VG Wort

VJPD

VJUSL

VKA

VOA

VÖT

VS

VSJ

V.S.J.u.S

VSL

V.S.u.K.

V.S.V.A.

VT

\section{WAK}

WAV

WCC

WKKG

WSF

ZSV
Verband deutschsprachiger Autoren in Amerika

Vereinigung europäischer Journalisten (IA)

Verband der Hermann-Löns-Kreise e. V. in Deutschland und Österreich (D)

Verband der Journalisten der DDR

Verband der katholischen Schriftsteller Österreichs

Vereinigung demokratischer und sozialistischer Künstler e. V.

Verband der deutschen Kritiker e. V. (D)

Verband deutschsprachiger Übersetzer literarischer und wissen-

schaftlicher Werke e. V. (D)

Verband der Film- und Fernsehschaffenden der DDR

Verband Fränkischer Schriftsteller e. V.

Verwertungsgesellschaft Bild-Kunst (D)

Verband der Geistig Schaffenden Österreichs

Verwertungsgesellschaft Wort vereinigt mit der Verwertungsgesel1schaft Wissenschaft (D)

Verband Junger Publizisten Deutschlands (D)

Vereinigung junger unbekannter Schweizer Literaten

Verband Kasseler Autoren

Vereinigung Oberschlesischer Autoren (D)

Verband Osterreichischer Textautoren

Verband deutscher Schriftsteller (D)

Verband der Schweizer Journalisten

Vereinigung Sozialistischer Journalisten und Schriftsteller Öster-

reichs

Verein Schweizerischer Literaturfreunde

Verein der Schriftstellerinnen und Künstlerinnen (A)

Verein Schweizerischer Volksbühne-Autoren

Verband der Theaterschaffenden der DDR

Werkstatt Arbeiterkultur (CH)

Westdeutscher Autorenverband e. V. (D)

World Cultural Council

Wilhelm-Kotzde-Kottenrodt-Gemeinde (D)

World Science Fiction (IA)

Zürcher Schriftsteller-Verband und Verband Ostschweizer Autoren 


\section{Verzeichnis der Schriftstellerinnen und Schriftsteller}


